Scheuer, O., McLaren B. M., Weinberger, A., \& Niebuhr, S. (2012). Scripting Discussions For Elaborative, Critical Interactions. In: S. A. Cerri, W. J. Clancey, G. Papadourakis, \& K. Panourgia (Eds.), Proceedings of the 11th International Conference on Intelligent Tutoring Systems (ITS-2012), LNCS 7315 (pp. 647-648 ). Springer, Berlin

\title{
Scripting Discussions For Elaborative, Critical Interactions
}

\author{
Oliver Scheuer ${ }^{1}$, Bruce M. McLaren ${ }^{1,2}$, Armin Weinberger ${ }^{1}$, Sabine Niebuhr $^{3}$ \\ ${ }^{1}$ Saarland University, Saarbrücken, Germany \\ ${ }^{2}$ Carnegie Mellon University, Pittsburgh, PA, U.S.A. \\ ${ }^{3}$ Clausthal University of Technology, Clausthal, Germany \\ \{o.scheuer@mx.uni-saarland.de\}
}

\begin{abstract}
Scripting collaborative argumentation can be effective in helping students understand multiple perspectives in complex, ill-defined domains. We have developed a web-based collaborative learning environment and a collaboration script to support students in discussing and analyzing controversial texts. We present a study in which we varied one element of the script to support critical, elaborative interactions, namely whether or not students take a proponent and/or critic role. Our results suggest that roles have a positive effect on the extent of knowledge elaboration in student discussions.

Keywords: computer-supported collaborative learning, collaboration scripts, argumentation, argument mapping
\end{abstract}

\section{Introduction}

It is widely recognized that critical thinking skills play an important role in today's information societies. During the past two decades many computer-based tools have been developed to support the acquisition of argumentation skills [2]. We introduce a web-based collaborative learning environment that supports students in creating and discussing argument diagrams, and a collaboration script to support students in using this environment to discuss conflicting texts. We present a study that investigates whether an additional script component, in which students take "proponent" and "critic" roles, could improve the quality of student discussions in terms of critical, elaborative interactions.

\section{Learning Environment and Collaboration Script}

LASAD is a highly configurable, web-based argument-diagramming environment that allows groups of students to represent arguments graphically in the form of box-andarrow diagrams. Boxes represent statements and links represent argumentative and rhetorical relations of different types (e.g., "support", "opposition", "related to"). Besides a shared diagramming workspace students can use a chat to communicate with one another.

The FACT-2 collaboration script ("Fostering Argumentation Through Conflicting Texts") has been developed to support critical, elaborative discussion in student dyads based on conflicting texts. It is based on distributed resources, that is, each student has 
exclusive access to one of two texts. Student activities are structured in four different phases: Students (1) model their texts in LASAD individually, (2) discuss, based on the diagram, aspects of each text with the partner, (3) discuss connections between the two texts, and (4) agree on a joint position and compose a joint reasoned conclusion. A previous version of the script is described in [3].

\section{Study}

A quasi-experimental study using a pretest-intervention-posttest design has been conducted. Both conditions used LASAD and the collaboration script described above. Opposing texts regarding climate change were used (thesis: "Developed countries have to cut their carbon emissions drastically"). For Treatment dyads an additional role script component was administered, in which students were instructed to act as proponent of their text and a constructive critic of their partner's text. A sentence opener interface [4] was used to provide support for the proponent and constructive critic roles. The Comparison group used a standard chat instead.

Participants were students at Saarland University and received a participation fee. The sample comprises 12 Treatment dyads (i.e., with role script) and 10 Comparison dyads (i.e., no role script). The overall study took about 3 hours; 1.5 hours of which were spent on the actual task. An analysis of questionnaire data indicated that the conditions did not differ significantly in terms of relevant entry characteristics.

We report on an analysis of chat protocols. Based on the Rainbow coding framework [1] we developed and validated a coding scheme with satisfactory result $(\kappa=.76)$. We distinguished three levels of argumentative elaboration. To assess the quality of each protocol we analyzed the amount of "High" elaboration moves (i.e., ones that cite, elaborate, question or criticize relevant contents). We found a nonsignificant trend $(p=.07)$ with large effect size $(d=0.82)$ in terms of "High" codes in favor of the Treatment group, a result in accordance with our hypothesis.

Acknowledgements: We would like to thank Christoph Fehige for advice and support. The German Research Foundation (DFG) provided funding for this research.

\section{$4 \quad$ References}

1. Baker, M., Andriessen, J., Lund, K., van Amelsvoort, M., \& Quignard, M. (2007). Rainbow: A Framework for Analyzing Computer-Mediated Pedagogical Debates. IJCSCL 2(2-3), 247-272.

2. Scheuer, O., Loll, F., Pinkwart, N., \& McLaren, B.M. (2010). Computer-Supported Argumentation: A Review of the State of the Art. IJCSCL 5(1), 43-102.

3. Scheuer, O, McLaren, B.M., Harrell, M., \& Weinberger, A. (2011). Scripting Collaboration: What Affects Does it Have on Student Argumentation? In: T. Hirashima et al. (Eds.), Proc. of ICCE-2011 (pp. 181-188). Asia-Pacific Soc. for Computers in Education.

4. Soller, A. (2001). Supporting social interaction in an intelligent collaborative learning system. IJAIED 12, 40-62. 